\title{
Effect of aquaporin 1 and 4 on masticatory muscles degeneration as a result of aging
}

\author{
Tumer $\mathrm{MK}^{1,2}$, Demir $\mathrm{M}^{3}$, Cicek $\mathrm{M}^{3}$ \\ Department of Oral and Maxillofacial Surgery, Faculty of Dentistry, Gaziosmanpasa University, \\ Tokat, Turkey.dr_kemaltumer@yahoo.com
}

\section{ABSTRACT}

OBJECTIVE: The changes in the mouth structures due to aging cause some structural and functional changes by affecting masticatory muscles over time. The aim of this study was to evaluate the aging-related histopathologic changes and immunohistochemically assessed aquaporin 1 and 4 expressions on masseter and temporal muscles. MATERIAL AND METHODS: 14 Balb/c white mice $(50-80 \mathrm{~g})$ were used in this study. Group I consisted of young animals (2-month-individuals) $(n=7)$ and Group II consisted of older animals (18-month-old) $(n=7)$. After routine histological follow-ups were made, tissues were stained immunohistochemically for aquaporin 1 and aquaporin 4 as well as with hematoxylin-eosin.

RESULTS: It was seen that while the masseter and temporalis muscle tissues showed a high immunoreactivity $(+++)$ for aquaporin 1 and 4 in young mice, they showed a weak immunoreactivity (+) for aquaporin 1 and 4 in old mice $(p=0.001)$. In the $\mathrm{H}$-score assessment, aquaporin 1 and 4 immunoreactivity was significantly higher in young mice than in old mice $(p=0.002)$.

CONCLUSIONS: Consequently, it was shown that degeneration of the masticatory muscles increased with aging and there was a decrease in intra- and intercellular exchange of substances because of changing aquaporin 1 and aquaporin 4 expressions (Tab. 2, Fig. 4, Ref. 20). Text in PDF www.elis.sk.

KEY WORDS: aquaporin 4, aquaporin 1, stomatognathic system, aging, masseter muscle.

\section{Introduction}

Aquaporins (AQP) are a family of membrane channel proteins acting as selective pores through which water can pass in plasma membranes of many tissue and cell types (1). AQPs are widely distributed and fulfill different functions in different tissues. The AQP family can be divided into two groups by considering the structural and functional differences. The members of Group 1 (AQP 0-2, 4-6 and 8) are primarily water selective channels. The members of Group 2 (AQP 3, 7, 9, and 10) are permeable to water as well as neutral soluble substances, including glycerol and urea (2).

It is known that the structure and function of skeletal muscle changes with aging (3). However, the changes related with age vary between skeletal muscle types (4). The masseter, genioglossus and geniohyoid muscles play an important role in chewing, swallowing and in the maintenance of the patency of the upper respiratory tract (5). Reports have been published on the changes related with age in rat muscle and it was stated that the perfor-

${ }^{1}$ Department of Oral and Maxillofacial Surgery, Faculty of Dentistry, Gaziosmanpasa University, Tokat, Turkey, ${ }^{2}$ Department of Medical Biology, Faculty of Medicine, Gaziosmanpasa University, Tokat, and ${ }^{3}$ Department of Anatomy, Faculty of Medicine, Kahramanmaras Sütcü ImamUniversity, Kahramanmaras, Turkey

Address for correspondence: M.K. Tumer, PhD, DMD, Department of Oral and Maxillofacial Surgery, Faculty of Dentistiry, University of Gaziosmanpasa, Tokat, Turkey.

Phone: +90.543.3199155, Fax: +90.356.2124225 mance of the masseter muscle was protected with aging (6). The structure and function of skeletal muscle vary with age, but these changes depend on the type of skeletal muscle (5).

The aim of this study was to evaluate the aging-related histopathologic changes on masseter and temporal muscles and detect the age-related alterations in aquaporin 1 and 4 expressions, immunohistochemically.

\section{Materials and methods}

\section{Supply of Animals}

After this study was approved by the local ethics committee (HADYEK-44), it was conducted at the Gaziosmanpasa University, Experimental Medicine Research Center. 14 Balb/c white mice $(50-80 \mathrm{~g})$ were used in this study. The animals were divided into two equal groups each containing seven animals. Group I consisted of young animals (2-month-old) $(\mathrm{n}=7)$ and Group II consisted of older animals (18-month-old) $(n=7)$. The animals were kept at room temperature $\left(22 \pm 1{ }^{\circ} \mathrm{C}\right)$ and $40-50 \%$ humidity before the application. Light level was set to cycle between 12-h light / 12-h dark. The animals were set free to eat and drink. The mice were kept under observation for 1 week and daily physical examination was performed.

\section{Obtaining samples}

The mice without any health problems were killed by the exsanguination method under anesthesia induced with ketamine/xy- 
lazine $(50 / 10 \mathrm{mg} / \mathrm{kg})$. The temporomandibular joints including the ramus of the mandible and a portion of the temporal bone of the mice were removed in a block to preserve the integrity. They were taken in $10 \%$ formalin solution for use in immunohistochemical and histopathological evaluations.

\section{Histological examination of the masseter and temporalis muscles}

The tissues were left in the formalin solution until the section could be taken. After routine histological follow-ups were made, tissues were embedded in paraffin. $4-5-\mu$ m-thick tissue sections were taken from the paraffin embedded tissues. Then, they were stained with Hematoxylin-eosin (H\&E). The stained sections were examined under the Zeiss Axio Lab A1 light microscope.

\section{Immunohistochemical examination of the masseter and tempo-} ralis muscles

4-5-mm thick sections, which were taken from paraffin blocks, were stained immunohistochemically for Aquaporin 1 (Rabbit polyclonal to Aquaporin 1, AB110186, Cambridge, UK) and Aquaporin 4 (Rabbit polyclonal to Aquaporin 4, Abcam, AB34710, UK). The stained slides were examined with the research microscope (Zeiss Axio Lab A1) and also, they were evaluated and photographed. Then immunohistochemical assay was performed using the H-score analysis (7). The intensity of aquaporin 1 and aquaporin 4 immunoreactivity was evaluated semi-quantitatively by using the categories of stain intensity during follow-up: 0 (No staining), $1+$ (Weak-but-detectable staining), 2+ (Moderate or significant staining), and 3+ (Intensive staining). Firstly, H-score value was obtained for each tissue by computing the sum of the percentages of the cells according to the categories of stain intensity. Then, this value was multiplied by the weighted intensity of staining by using the H-score formula $=\Sigma \mathrm{Pi}(\mathrm{i} 1+)$ (" $i$ " representing the intensity scores and "Pi" representing the relative percentage of cells). Each slide was evaluated under light microscope (in 40X magnification). The percentage of the cells in each intensity in these areas was identified at different times by two researchers

Tab. 1. Comparison of the masticatory muscles of young and old mice.

\begin{tabular}{lccccc}
\hline Group & Young $(\mathrm{n}=7)$ & Old $(\mathrm{n}=7)$ & \multirow{2}{*}{$\mathrm{t}$} & $\mathrm{p}$ \\
\cline { 1 - 3 } Masticatory Muscles & $\bar{x} \pm S D$ & $\bar{x} \pm S D$ & & & \\
\hline Temporalis AQP 1 & $164.29 \pm 22.42$ & $108.14 \pm 6.41$ & 6.370 & $0.001^{*}$ \\
Temporalis AQP 4 & $151.71 \pm 18.98$ & $102.29 \pm 12.79$ & & 5.714 & $0.001^{*}$ \\
Masseter AQP 1 & $189.29 \pm 15.40$ & $131.14 \pm 18.86$ & 6.317 & $0.001^{*}$ \\
Masseter AQP 4 & $177.71 \pm 18.89$ & $107.00 \pm 17.51$ & 7.263 & $0.001^{*}$ \\
\hline
\end{tabular}

* Difference is statistically significant; $\alpha=0.05$ : independent samples $t$ test who were unaware of the species and source of the tissues. The average scores of both researchers were used.

\section{Statistical analysis}

The Shapiro-Wilk test was used for testing normality. The data were normally distributed. $\mathrm{p}$ values of $<0.05$ were considered to be significant at statistical level. The independent two-sample ttest was used to compare the groups.

\section{Results}

\section{Histological findings}

Hematoxylin-eosin staining findings

Histological structure of the masseter and temporalis muscles was photographed by a light microscope in young and old animals (Fig. 1). Degeneration areas were seen in muscle fibers due to aging. It was seen that myocyte proliferation and expansion in the intramuscular areas were greater in old mice compared to young mice. There was an increase in the number of necrotic muscle fibers and a decrease in the number of muscle cells with centrally located nucleus. Moreover, degenerative disorders and muscular dystrophies occurred in muscle fibers due to aging. It was seen that muscle fibers of young mice exhibited a healthier appearance than that of old mice (a low amount of both necrotic and angular fibers). In young mice, the excessive viability of the myofibers was disrupted and the number of them declined with aging. Large nuclei, small cytoplasms and large cell areas were seen in muscle fibers of young mice; however, it was noted that small nuclei, large cytoplasms and small cell areas were seen in muscle fibers of old mice. It was observed that age-related decline and shrinkage occurred in blood vessels.

\section{Immunohistochemical findings}

Immunohistochemical staining for aquaporin 1 and aquaporin 4 was performed in tissue sections of the masseter and temporalis muscles from the masticatory muscles of young and old mice. No immunoreactivity was observed in the negative control staining. The results were evaluated semi-quantitatively and are given in Table 2. It was seen that while the masseter and temporalis muscle tissues showed a high immunoreactivity $(+++)$ for aquaporin 1 and 4 in young mice (Figs 2 and 3), the masseter and temporalis muscle tissues showed a weak immunoreactivity $(+)$ for aquaporin 1 and 4 in old mice $(\mathrm{p}=0.001)$. In the $\mathrm{H}$-scoring results performed by immune positive staining of muscle cells in the transverse sec-

Tab. 2. Comparison of immunoreactivity concentrations of the masticatory muscles of young and old mice.

\begin{tabular}{|c|c|c|c|c|c|c|}
\hline & \multicolumn{2}{|c|}{ Young } & \multicolumn{2}{|c|}{ Old } & \multirow[b]{2}{*}{ Total n } & \multirow[b]{2}{*}{$\mathrm{p}$} \\
\hline & $\mathrm{n}$ & $\begin{array}{c}\text { Immune reactivity } \\
\text { concentration }\end{array}$ & $\mathrm{n}$ & $\begin{array}{c}\text { Immune reactivity } \\
\text { concentration }\end{array}$ & & \\
\hline Temporalis Aqp 1 & 7 & +++ & 7 & + & 14 & $0.001^{*}$ \\
\hline Temporalis Aqp 4 & 7 & +++ & 7 & + & 14 & $0.001 *$ \\
\hline Masseter Aqp 1 & 7 & +++ & 7 & + & 14 & $0.001 *$ \\
\hline Masseter Aqp 4 & 7 & +++ & 7 & + & 14 & $0.001 *$ \\
\hline
\end{tabular}

* statistically Significant; $\alpha=0.05$; Fisher Exact test 

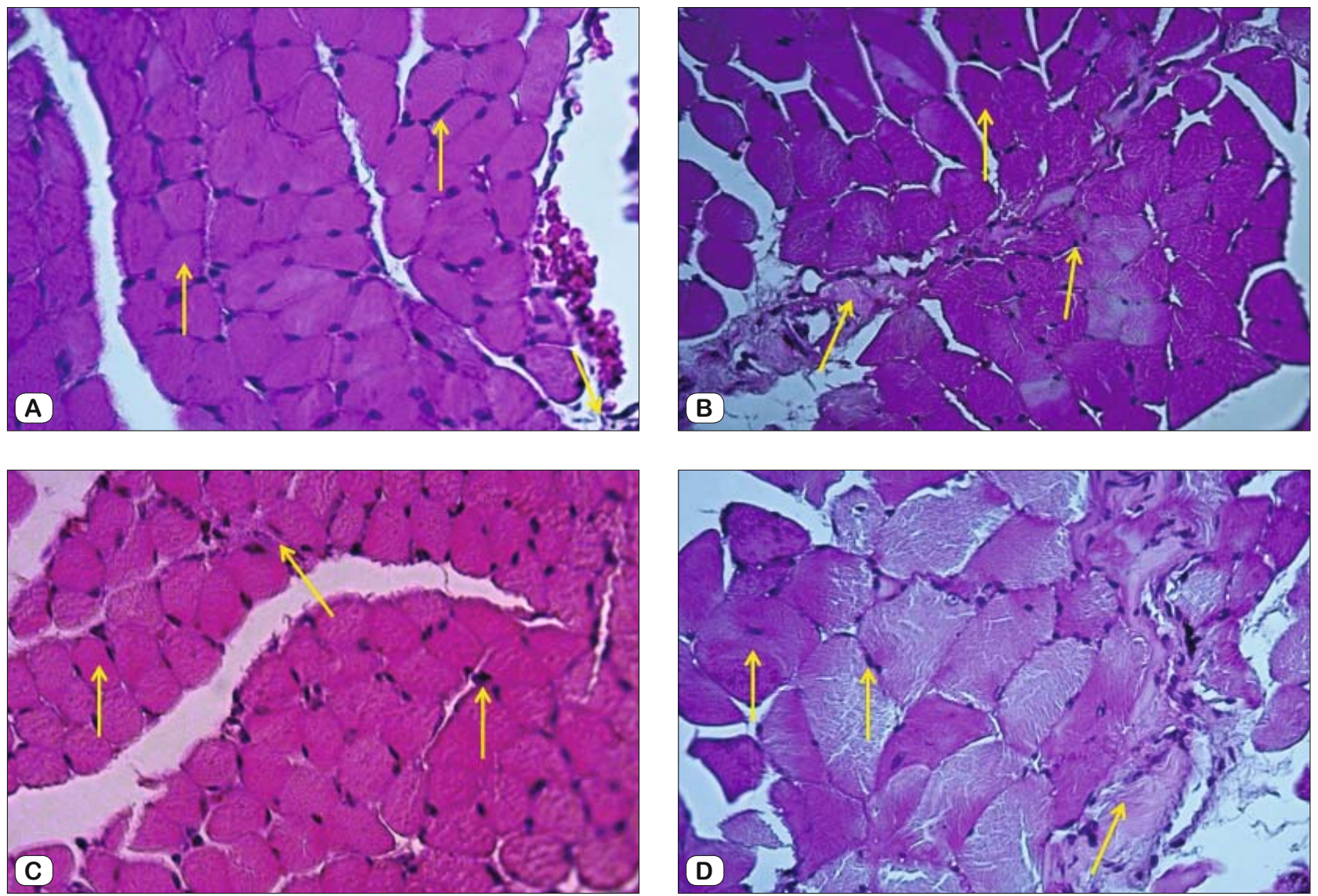

Fig. 1. Hematoxylin-eosin staining, 40X. A) Young mice, masseter muscle, B) Old mice, masseter muscle, C) Young mice, temporal muscle, D) Old mice, temporalis muscle.

tions of the masseter and temporalis muscles forming musculi masticatorii, aquaporin 1 and aquaporin 4 immunoreactivity was higher at a significant level in young mice than in old mice ( $\mathrm{p}=$ 0.001) (Tab. 1).

\section{Discussion}

The physiological function of the chewing system is the chewing function. Functional structures such as teeth, supporting dental tissues, jaws, joints, muscles and vascular-nervous system constitute the chewing system. The morphology of the masticatory muscles must be complete for an effective chewing function. Therefore, we tried to analyze the aging-related changes in the masseter and temporalis muscles in this study. The adult mouse masticatory muscles contain only fast-muscle fibers (8). Many studies have reported that there were time-dependent changes in the characteristics of muscle fibers in fetal masseter muscle and postnatal mouse tongue muscle (9). Shida et al (10) showed that the chewing muscles differed according to superficial and deeplayer muscle fiber properties together with the muscle fibers moving in different directions. In our study, degeneration areas were seen in muscle fibers due to aging as a result of histopathological analysis. It was seen that myocyte proliferation and expansion in the intramuscular areas were greater in old mice compared to young mice. There was an increase in the number of necrotic muscle fibers and a decrease in the number of muscle cells with centrally located nucleus. Moreover, degenerative disorders and muscular dystrophies occurred in muscle fibers due to aging. It was seen that muscle fibers of young mice exhibited a healthier appearance than that of old mice (a low amount of both necrotic and angular fibers). In young mice, the excessive viability of the myofibers was disrupted and the number of them declined with aging. Large nuclei, small cytoplasms and large cell areas were seen in muscle fibers of young mice; however, it was noted that small nuclei, large cytoplasms and small cell areas were seen in muscle fibers of old mice. It was observed that age-related decline and shrinkage occurred in blood vessels.

Aquaporins are a family of hydrophobic membrane channel proteins expressed in various types of cells in various organ systems, especially in epithelial and endothelial cells. Aquaporins act as selective pores that provide bidirectional water-passage through biological membranes, and thereby they allow the regulation of osmotic homeostasis in relation to the movement of melted material and extracellular medium (2). In particular, aquaporin 4 (AQP4) is the water channel that is found in mammalian skeletal muscle in vivo (11). Previous studies on AQP expression in the muscles 

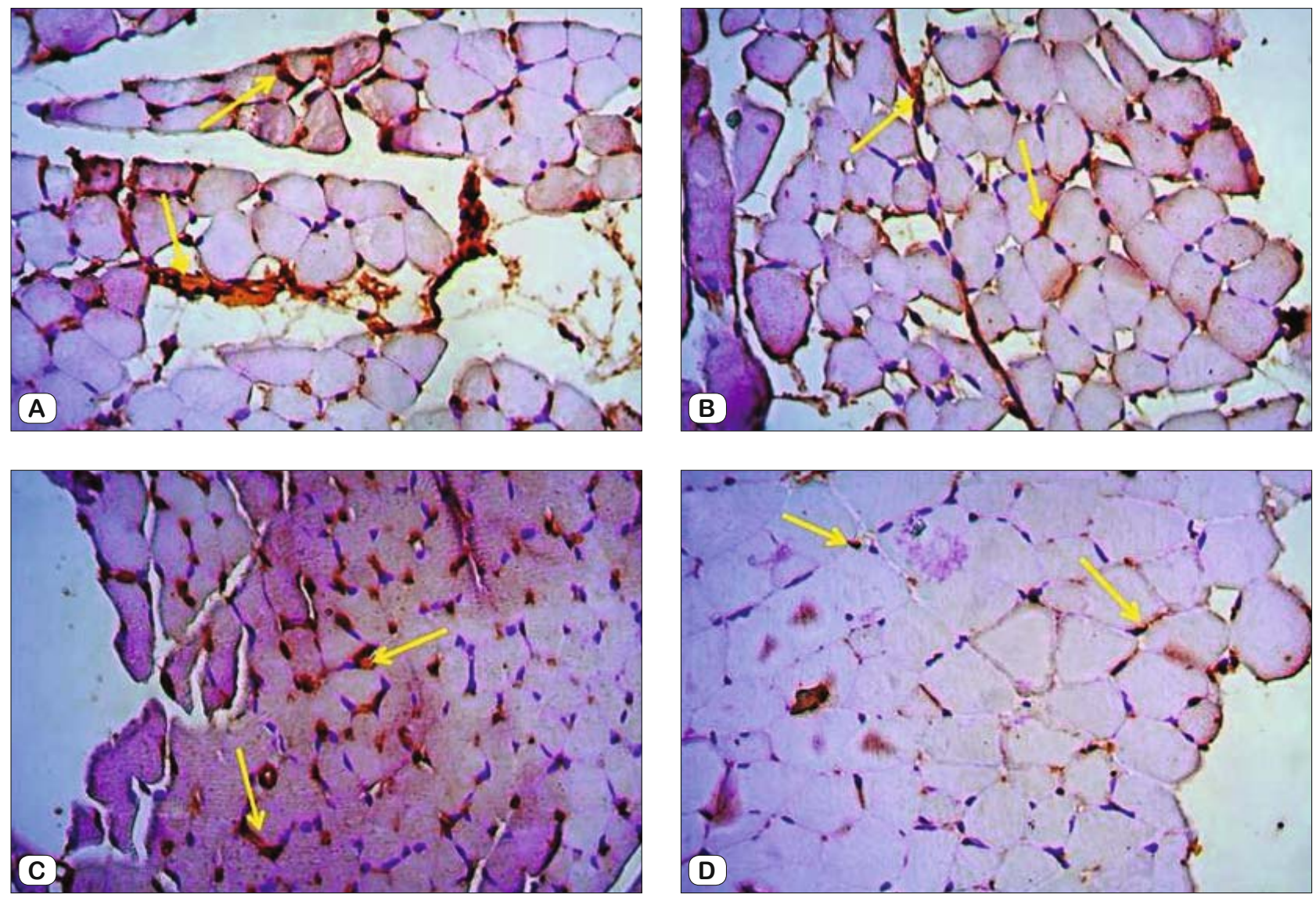

Fig. 2. Immunohistochemical staining of Aquaporin 1, 40X. E) Young mice, masseter muscle, F) Old mice, masseter muscle, G) Young mice, temporal muscle, $\mathrm{H}$ ) Old mice, temporalis muscle.

focused primarily on AQP1 and AQP4. As a result of immunohistochemical staining that we performed in our study, it was seen that while the masseter and temporalis muscle tissues showed a high immunoreactivity $(3+)$ for AQP1 and AQP4 in young mice, the masseter and temporalis muscle tissues showed a weak immunoreactivity $(1+)$ for $\mathrm{AQP} 1$ in old mice. This situation demonstrates that AQP1 and AQP4 in both young and old mice are found in the physiology of the masticatory muscles and play in the activity of the masticatory muscles.

AQP4 mediates water transport between blood and myofibrils for the modulation of rapid volume changes during muscle contraction and is expressed in microvessels together with AQP1 (12). It has also been suggested that AQP4 is involved in the regulation of myofiber energy metabolism in addition to modulating the osmotic pressure in myofibrils (11). On the other hand, pathological findings such as edema were not detected in skeletal muscle morphology in transgenic mice that overexpressed AQP4 (13). It was reported that muscle strength, osmotic water permeability and water content of skeletal muscle did not change in AQP4 knockout mice compared to wild-type mice (14). Therefore, there is no consensus on the physiological role of AQP4 in protecting skeletal muscle homeostasis in vivo (15). As a result of the H-score analysis in our study, it was seen that the amount of AQP4-positive cells was higher in the masticatory muscles of young mice but was significantly decreased in the masticatory muscles of old mice. Intensive staining $(3+)$ of AQP4 in the masticatory muscles of young mice suggests that the exchange of substances between extracellular medium and intracellular medium through the water channels in the cell membrane is greater. However, we think that weak staining $(1+)$ of AQP4 in the masticatory muscles of old mice indicates that the number of the water channels involved in the exchange of substances is reduced due to aging and therefore this may cause disruption of homeostasis. We assume that high expression of AQP4 in young mice provides that osmotically active metabolites are released more easily into myoplasm at higher activation levels of the chewing muscles during contraction.

In addition to AQP4, there is evidence for the expression of other AQPs in human skeletal muscle. Süleymanyan and Baumgarten (16), 1996 used membrane hydraulic conductivity to measure the amount of net water-movement across the sarcolemma of rabbit ventricular myocytes in order to explore the functional significance of AQPs. They concluded that the values were significantly higher significant than the expected value for AQP1 alone. Alternatively, Kellen and Bassingthwaighte (17), 2003 reported that 

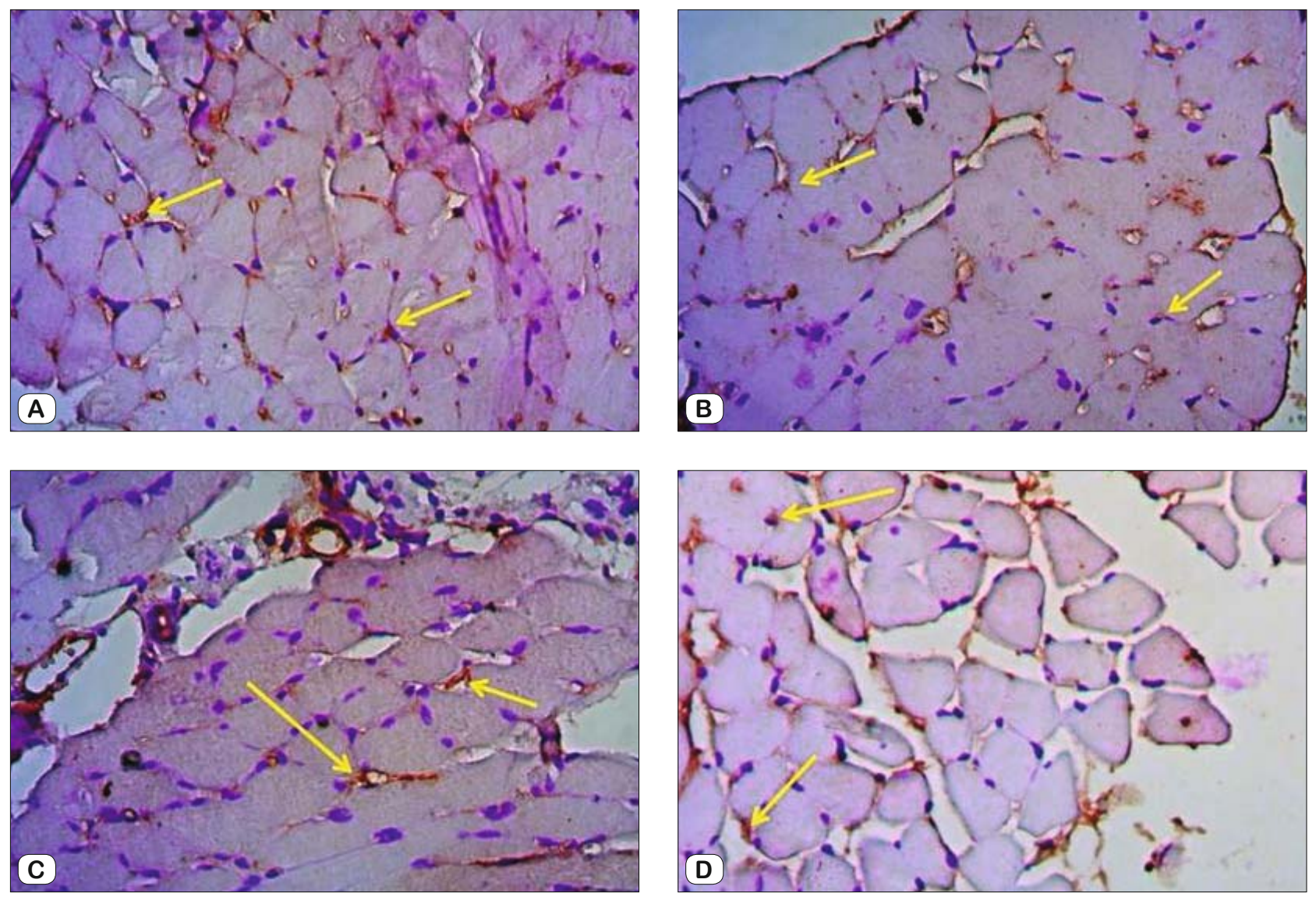

Fig. 3. Immunohistochemical staining of Aquaporin 4, 40X. I) Young mice, masseter muscle, J) Old mice, masseter muscle, K) Young mice, temporal muscle, L) Old mice, temporalis muscle.

$28 \%$ of the transcapillary water-flux occurred via transepithelial transport (through AQPs) during steady-state conditions and the remaining amount passed through interendothelial clefts. In the light of our findings, the fact that the amount of AQP1 positive cells was higher at a significant level in young mice than in older mice suggests that it is associated with endothelial cell migration and vascular growth, and the fact that the amount of AQP1 positive cells was lower at a significant level in old mice than in young mice suggests that young mice have more endothelial regenerative capacity. Moreover, we suggest that the AQP1 pool is present in myocytes with endothelial capillaries in the chewing muscles. The changes in AQP1 expression in myocytes may show the functional significance of the AQP1 protein. Understanding how water is regulated in AQP channels in the physiological and pathological conditions in myocytes with aging can provide the development of therapeutic strategies aimed at preventing edema in the muscle or facilitating recovery after injuries such as ischemia and reperfusion.

It has been reported that $\mathrm{AQP} 1$ is rich in microvascular endothelium, including the capillary endothelium in the skeletal muscle. The localization of AQP1 in the sarcolemma of human skeletal muscle fibers has also been demonstrated (18). In humans, the cross-sectional area and density of the masseter and temporalis muscles decrease as a result of aging (19). Electromyographic (EMG) records in these muscles showed age-related physiological changes which included a reduction in muscle activity (20). Previous studies in humans have indicated a change in chewing function together with a greater number of bites required for mouth movements and bolus formation because of the underlying changes in muscle morphology and physiology with increasing age (19). As a result of the H-Score analysis in our study, it was seen that the amount of AQP4 positive cells was higher in the masticatory muscles of young mice but was significantly decreased in the masticatory muscles of old mice. Intensive staining (3+) of AQP4 in the masticatory muscles of young mice suggests that the exchange of substances between extracellular medium and intracellular medium through the water channels in the cell membrane is greater. However, we think that weak staining (1+) of AQP4 in the masticatory muscles of old mice indicates that the number of the water channels involved in the exchange of substances is reduced due to aging and therefore this may cause disruption of homeostasis. It was seen that AQP1 and AQP4 protein levels were decreased as a result of muscle loss with aging. In addition, there was a decrease in the expression levels of AQP1 and AQP4 in endothelium and 
$669-675$
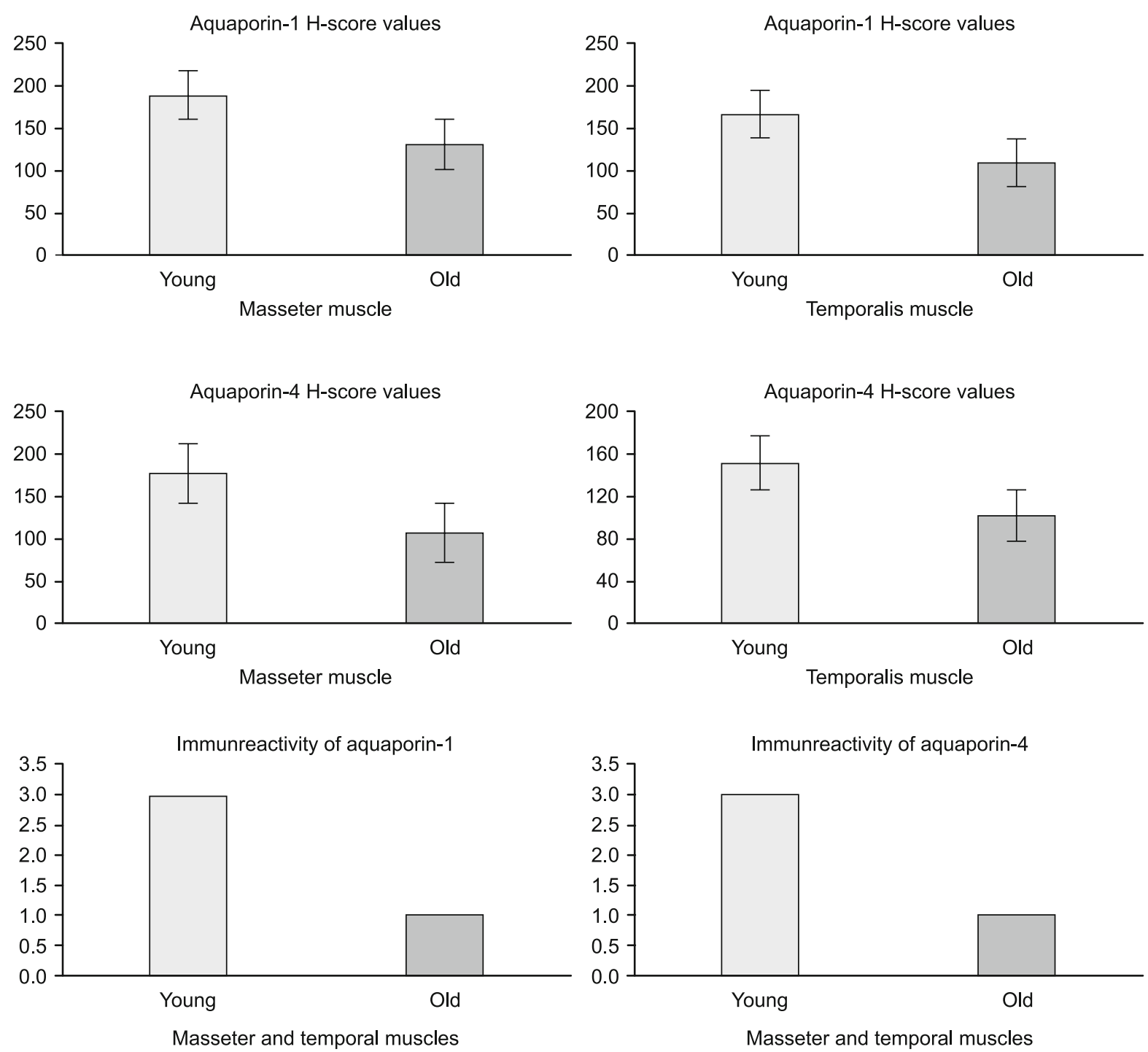

Fig. 4. Comparison of $\mathbf{H}$-scores and immunoreactivity of aquaporin 1 and aquaporin 4 in the masticatory muscles of young and old mice.

myofibrils of the chewing muscles due to aging. We think that this reduction is related to the fact that the chewing muscles are among the fastest contracting muscles in the body and the fact that osmotic permeability decreases in muscle sarcolemma with aging (Fig. 4).

Consequently, degeneration of myocytes and myofibrils of the chewing muscles and loss of muscle mass (i.e. muscular dystrophy) increase with aging. There is a decrease in the exchange of substances between intercellular medium and intracellular medium. Functional impairments in the cell membrane together with the negative changes in the number and structure of the water channels located in the cell membrane can cause irreversible damage. In this context, we believe that it will be a guide in the investigation of new treatment methods for the removal of natural or operative damage due to aging.

\section{References}

1. King LS, Agre P. Pathophysiology of the aquaporin water channels. Annu Rev Physiol 1996; 58: 619-648.

2. Wang W, Hart PS, Piesco NP, Lu X, Gorry MC, Hart TC. Aquaporin expression in developing human teeth and selected orofacial tissues. Calcif Tissue Int 2003; 72: 222-227.

3. Degens H. Age-related skeletal muscle dysfunction: causes and mechanisms. J Musculoskelet Neuronal Interact 2007; 7: 246-252.

4. Imagita H, Yamano S, Tobimatsu Y, Miyata H. Age-related changes in contraction and relaxation of rat diaphragm. Biomed Res 2009; 30 : $337-342$.

5. Kaneko S, lida RH, Suga T, Morito M, Yamane A. Age-related changes in rat genioglossus, geniohyoid and masseter muscles. Gerodontology 2014; 31 (Suppl 1): 56-62. 
6. Norton M, Verstegeden A, Maxwell LC, McCarter RM. Constancy of masseter muscle structure and function with age in F344 rats. Arch Oral Biol 2001; 46: 139-146.

7. Kanter M. Protective effects of thymoquinone on the neuronal injury in frontal cortex after chronic Toluene exposure. J Mol Histol 2011; 42: 39-46.

8. Eason JM, Schwartz GA, Pavlath GK, English AW. Sexually dimorphic expression of myosin heavy chains in the adult mouse masseter. $\mathrm{J}$ Appl Physiol 2000; 89: 251-258.

9. Usami A, Abe S, Ide Y. Myosin heavy chain isoforms of the murine masseter muscle during pre- and post-natal development. Anat Histol Embryol 2003; 32: 244-248.

10. Shida T, Abe S, Sakiyama K et al. Superficial and deep layer musclefiber properties of the mouse masseter before and after weaning. Arch Oral Biol 2005; 50: 65-71.

11. Ishido M, Nakamura T. Aquaporin 4 Protein Is Stably Maintained in the Hypertrophied Muscles by Functional Overload. Acta Histochem Cytochem 2009; 49 (Suppl 3): 89-95.

12. Frigeri A, Nicchia GP, Balena R, Nico B, Svelto M. Aquaporins in skeletal muscle: reassessment of the functional role of aquaporin 4. FASEB J 2004; 18: 905-907.

13. Wakayama Y, Takahashi J, Shibuya S et al. Generation of muscle aquaporin 4 overexpressing transgenic mouse: its characterization at
RNA and protein levels including freeze-fracture study. Micron 2007; 38: $257-267$.

14. Yang B, Verbavatz JM, Song $Y$ et al. Skeletal muscle function and water permeability in aquaporin 4 deficient mice. Am J Physiol Cell Physiol 2009; 278: 1108-1115.

15. Kaakinen M, Salmela P, Zelenin S, Metsikko K. Distribution of aquaporin 4 on sarcolemma of fast-twitch skeletal myofibres. Cell Tissue Res 2007; 329: 529-539.

16. Suleymanian MA, Baumgarten CM. Osmotic gradient-induced water permeation across the sarcolemma of rabbit ventricular myocytes. J Gen Physiol1996; 107: 503-514.

17. Kellen MR, Bassingthwaighte JB. Transient transcapillary exchange of water driven by osmotic forces in the heart. Am J Physiol Heart Circ Physiol 2003; 235: 1317-1331.

18. Au CG, Butler TL, Egan JR, et al. Changes in skeletal muscle expression of AQP1 and AQP4 in dystrophinopathy and dysferlinopathy patients. Acta Neuropathol 2008; 116 (Suppl 3): 235-246.

19. Krekeler BN, Connor NP. Age-related changes in mastication are not improved by tongue exercise in a rat model. Laryngoscope 2016; 127 (Suppl 1): E29-E34

20. Cecilio FA, Regalo SC, Palinkas $M$ et al. Ageing and surface EMG activity patterns of masticatory muscles. J Oral Rehabil 2010; 37: 248-255.

Received June 13, 2017. Accepted July 5, 2017. 